\title{
Corrigendum to Integral points on Abelian varieties
}

\author{
Invent. Math. 81, 341-346 (1985)
}

Joseph H. Silverman

Massachusetts Institute of Technology. Cambridge, MA 02139, USA

The statement of Theorem 1 in the above paper should read as follows.

Theorem 1. Let $k$ be a number field and $J / k$ the Jacobian variety of a curve $C / k$ of genus $g \geqq 2$. There exists an ample, effective, irreducible divisor $D \in \operatorname{Div}(J)$ with the following property:

Let $K / k$ be a finite extension, $R_{S}$ the ring of $S$-integers in $K$ for some finite set of places $S$ of $K, n$ an integer, and $f_{0}, \ldots, f_{r} \in \Gamma\left(A, \mathfrak{O}_{A}(n D)\right)$ functions such that the map $\left[f_{0}, \ldots, f_{r}\right]: A \rightarrow \mathbb{P}^{r}$ is an embedding. Then the set

$$
\left\{P \in J(K): f_{i} \text { is regular at } P \text { and } f_{i}(P) \in R_{S} \text { for all } 0 \leqq i \leqq r\right\}
$$

is finite.

The published proof of Theorem 1 uses the assertion that if a function $f \in K(J)$ has a pole along $D$, and if $f(P) \in R_{S}$, then for any $v \notin S$, the reduction of $P$ (modulo $v$ ) does not lie on the support of $D$ (modulo $v$ ). This assertion is incorrect; it may fail if $P$ (modulo $v$ ) lies on the intersection of the polar divisor of $f$ and the zero divisor of $f$ (modulo $v$ ). [I.e. If the rational map $f: J \rightarrow \mathbb{P}^{1}$ (taken modulo $v$ ) is not defined at $P$ (modulo $v$ ).]

This problem is eliminated in the formulation given above. By requiring that $\left[f_{0}, \ldots, f_{r}\right]: J \rightarrow \mathbb{P}^{r}$ be an embedding, and that $f_{i}(P) \in R_{S}$ for all $0 \leqq i \leqq r$, it follows that $P$ (modulo $v$ ) does not lie on the support of $n D$ (modulo $v$ ) for all $v \notin S$. (Note that it may be necessary to enlarge the set $S$ by finitely many places so that $\left[f_{0}, \ldots, f_{r}\right]: J \rightarrow \mathbb{P}^{r}$ is an embedding (modulo $v$ ) for all $v \notin S$. This does not affect the remainder of the argument.) 